\title{
Mathematical study of very high voltage power networks I: The optimal DC power flow problem
}

J. Frédéric BONNANS

\section{$\mathbf{N}^{\circ} 2408$}

Décembre 1994

PROGRAMME 5

Traitement du signal,

automatique

et productique

\section{apport \\ de recherche}





\title{
Mathematical study of very high voltage power networks I: The optimal DC power flow problem
}

\author{
J. Frédéric BONNANS* \\ Programme 5 - Traitement du signal, automatique et productique \\ Projet Programmation mathématique \\ Rapport de recherche $\mathrm{n}^{\circ} 2408$ - Décembre 1994 - 15 pages
}

\begin{abstract}
The optimal power flow problem consists in setting the voltage and power delivered at the nodes of an electrical network, in order to minimize the loss of power over the lines. This paper is the first of a series dedicated to the mathematical study of this problem, based on an asymptotic analysis, in which the small parameter is the inverse of the reference voltage of the network. We call this scheme the very high voltage approximation. Here we deal with the case of direct current. We obtain an analytic expansion for the optimal value and the solution.
\end{abstract}

Key-words: Electrical networks, nonlinear optimization, asymptotic analysis, sensitivity analysis, expansion of solutions, directional constraint qualification.

(Résumé : tsvp)

* Email: Frederic.Bonnans@inria.fr. This study was supported by Electricité de France. Thanks are due to G. Blanchon and J.C. Dodu, from Direction des Etudes et Recherches, EDF, for the numerous discussions I have had with them for many years.

Unité de recherche INRIA Rocquencourt

Domaine de Voluceau, Rocquencourt, BP 105, 78153 LE CHESNAY Cedex (France)

Téléphone : (33 1) 39635511 - Télécopie : (33 1) 39635330 


\section{Étude mathématique des réseaux électriques de très haute tension I : Le problème du flot de puissance optimal en courant continu}

Résumé : Le problème du flot de puissance optimal consiste à fixer les tensions et puissances fournies aux différents noeuds d'un réseau électrique, de façon à minimiser la somme des pertes sur les différentes lignes. Cet article est le premier d'une série consacrée à l'étude mathématique de ce problème, basée sur une analyse asymptotique, dans laquelle le petit paramètre est l'inverse de la tension de référence du réseau. Ce schéma est appelé approximation des très hautes tensions. Le présent article traite de réseaux à courant continu. Nous obtenons le développement du coût optimal et de la solution du problème.

Mots-clé : Réseaux électriques, optimisation non linéaire, analyse asymptotique, analyse de sensibilité, développement de solutions, qualification directionnelle des contraintes. 
AMS subject classifications. 49K40, 90C31, 58C15

1. Introduction. The optimal power flow problem is an important issue, which consists in setting in an optimal way the voltage and power delivered at the nodes of an alternative current (AC) network. The distribution of voltage and power is subject to certain bounds, and must comply with Kirchhoff's and Ohm's law. A typical criterion is to minimize the loss of energy over the network (see e.g. [3]).

This paper is the first of a series dedicated to the mathematical study of this problem, based on an asymptotic analysis, in which the small parameter is, roughly speaking, the inverse of the nominal voltage of the network. We call this scheme the high voltage approximation. This choice of a small parameter is natural, as industrial networks use indeed very high values for the voltage. The approximation scheme gives a considerable insight into the problem, as the limit problem (which, after of course a proper scaling, is well defined) has its active and reactive parts decoupled. This means that for a sufficiently high voltage, the coupling between active and reactive parts is weak, a property that can be very useful for numerical purposes. Indeed, on the basis of our perturbation analysis, one can prove the rapid convergence of some algorithms with decoupled equations (actually, this was the motivation of our study). These algorithms can be used for solving the power flow problem (without optimization) as well as for the optimal power flow problem.

The main mathematical tool of these papers is the perturbation theory for nonlinear programming, a subject in which significant progress was made in the last few years, e.g. $[2],[6],[10],[15]$. Indeed, this study can be viewed as a real-world application of the abovementioned theory.

While the application deals with alternative current, it is useful to consider first the analogous problem with direct current. This allows to study a problem with much simpler equations, which nevertheless retains some of the flavor of the real application. Even for readers whose primary interest lies only in real world applications, it is advisable to read this paper first in order to get accustomed with some basic tools of the perturbation theory for nonlinear programming, whereas mathematicians will be pleased, as always, to deal with a simplified model that allows a complete mathematical discussion.

The other parts of this study are devoted to AC networks. In part II, we discuss the power flow problem (without optimization), for which an early reference is [1]. we use the high voltage approximation, in which we assume that the real part of impedances is small; the phase of the limit problem is shown to be constant over the network, while the voltage is solution of a linear "direct current" equation. We discuss the algorithmic consequences of this fact. We also show that, when the resistances are negligible, the equations derive from a potential, which has a closed form expression. We also discuss the constant active power flow approximation. While in general the active power flow has no reason to be constant, we show that in the framework of the high voltage approximation, the active power flow has little dependence with respect to the input of reactive power, as observed on real-world networks in the pioneering paper of [8]. Again, when the resistances are negligible, we show that the relation between voltage and input of reactive power derives from a potential, whose 
expression is still in closed form, albeit more involved. Also important are the algorithmic consequences, which are a mathematical justification of the methods proposed by [8].

In part III we obtain our final results, namely the expansion of solutions, by applying the perturbation theory for nonlinear programming to the AC optimal power flow problem, in the framework of the high voltage approximation.

The present paper is structured as follows. In section 2, we recall the equation of the direct current power network, and state the problem of minimizing the loss of power over the network. Section 3 introduces the high voltage approximation. We show that, after a proper scaling, the limit problem is well-posed, and exhibit its solution. In section 4, we recall the mathematical tools from the perturbation theory for nonlinear programming that we apply, namely the results by [2] and [7]. We actually combine these two results in order to state a third one. Section 5 is devoted to the analysis of the limit problem. We note that the limiting problem is non-qualified, although there exist multipliers associated with the solution. In section 6 , we obtain the analytic expansion for the optimal value and the solution. We ultimately give physical interpretations of the expansion of the solution in section 7 .

\section{Table of notations}

$F(\mathcal{P})$ set of feasible points of an optimization problem $(\mathcal{P})$,

$S(\mathcal{P})$ set of solutions of an optimization problem $(\mathcal{P})$,

$v(\mathcal{P})$ optimal value of an optimization problem $(\mathcal{P})$,

$\mathcal{S}$ set of nodes of the network, numbered from 1 to $n$, excluding the reference node

0 ,

$n=|\mathcal{S}|$ cardinal of $\mathcal{S}$,

$Y_{k l}$ admittance between nodes $k$ and $l$,

$I_{k l}$ current from node $k$ to node $l$,

$V_{k}$ voltage at node $k$,

$V^{R}$ voltage at reference node,

$J_{k}$ input of current at node $k$,

$P_{k}$ input of power at node $k ; P_{k}:=V_{k} J_{k}$,

$Z$ admittance matrix.

2. Setting of the optimal direct current power flow problem. We consider a network composed of passive elements, namely resistances, with a possible injection of currents at the nodes. The voltages and currents are subject to Ohm's and Kirchhoff's law, and we state formally the problem of minimizing the energy losses, while respecting some bound constraints on the voltages and injection of power at the nodes. In the discussion below, we use some of the definitions given in the table of notations. We may write Ohm's law as

$$
I_{k l}=Y_{k l}\left(V_{k}-V_{l}\right),
$$

where $V \in \mathbb{R}^{n}$ is the vector of voltages. The matrix $Y$ is symmetric with a null diagonal, and nonnegative elements. We may interpret a null value of $Y_{k l}$ as the absence of line between 
the nodes $k$ and $l$. The current $J_{k}$ injected at node $k$ satisfies Kirchhoff's law

$$
J_{k}=\sum_{l} I_{k l}=\sum_{l} Y_{k l}\left(V_{k}-V_{l}\right)
$$

Thus the power injected at node $k$ is

$$
P_{k}:=J_{k} V_{k}=V_{k} \sum_{l} Y_{k l}\left(V_{k}-V_{l}\right) .
$$

Whenever $V_{k} \neq 0$, equation (3) is equivalent to

$$
\sum_{l} Y_{k l}\left(V_{k}-V_{l}\right)-P_{k} / V_{k}=0 .
$$

Let $Z$ be the $n \times n$ impedance matrix, defined by

$$
Z_{k k}:=\sum_{l} Y_{k l} ; \quad Z_{k l}:=-Y_{k l}, \quad k \neq l .
$$

We note that $Z$ is semi-positive definite, as

$$
V^{t} Z V=\sum_{\substack{k, l \\ k \neq l}} Y_{k l}\left(V_{k}-V_{l}\right)^{2} \geq 0
$$

is the sum of loss of power over all lines. We may write (4) as ${ }^{1}$

$$
Z V-P / V=0 .
$$

We will refer to (5) as the power equation. One may find in [4] a study of equation (5) in which the value of either $V$ or $P$ is given at each node. One of the results of [4] is that (5) may have multiple solutions. For instance, denoting by $\mathcal{S}_{P}$ the set of nodes over which $P$ is fixed, when $P>0$ over $\mathcal{S}_{P}$ and $\mathcal{S}_{P} \stackrel{\neq}{\subset} \mathcal{S}$, then there exist exactly $2^{\left|\mathcal{S}_{P}\right|}$ solutions, each of them being associated with a convention of sign of the components of $V$ over $\mathcal{S}_{P}$. In the sequel, we limit ourself to the study of the solutions close to a certain nominal value.

We consider the problem of minimization of losses

$$
\operatorname{Min}_{V, P} \frac{1}{2} V^{t} Z V ; Z V-P / V=0 ; V_{0}=V^{R} ; V^{b} \leq V \leq V^{\sharp} ; P^{b} \leq P \leq P^{\sharp} .
$$

In this problem $V^{R} \in \mathbb{R}_{+*}$ (the set of positive real numbers) is the reference value for the voltage, and $V^{b}, V^{\sharp}, P^{b}$ and $P^{\sharp}$ are given $n$-dimensional vectors that satisfy $V^{b} \leq V^{\sharp}$ and $P^{b} \leq P^{\sharp}$. The components of the lower bounds $V^{b}$ and $P^{b}$ have values in $\mathbb{R} \cup\{-\infty\}$,

\footnotetext{
${ }^{1}$ the division of vectors, as well as their multiplication, is to be understood componentwise.
} 
whereas those of the upper bounds $P^{\sharp}$ and $V^{\sharp}$ have values in $\mathbb{R} \cup\{\infty\}$. An infinite value simply means an absence of lower or upper bound constraint.

It seems not easy to determine if the above problem is well posed, and stable with respect to perturbations. First, the power equation is not itself well posed, unless we make specific assumptions on the data. In addition, some conditions on the bounds should be added, in order to make these bounds compatible with the power equation. We conclude that, to be able to conduct an analysis of this problem, we have to make some assumptions. Because some of the real-world networks have very high voltage values, a natural possibility is to consider the inverse of the reference value of the voltage as a small parameter, and to let this small parameter go to zero.

3. The very high voltage approximation. We introduce the very high voltage approximation by embedding $(\mathcal{P})$ in the family of problems

$$
\operatorname{Min}_{V, P} \frac{1}{2} V^{t} Z V ; Z V-P / V=0 ; V_{0}=\frac{V^{R}}{\sqrt{\varepsilon}} ; \frac{V^{b}}{\sqrt{\varepsilon}} \leq V \leq \frac{V^{\sharp}}{\sqrt{\varepsilon}} ; P^{b} \leq P \leq P^{\sharp},
$$

where $\varepsilon>0$ is a small parameter. We assume that $V^{R}>0$. Our aim is to compute an asymptotic expansion of the solution of the above problem when $\varepsilon \rightarrow 0$ (we will see in a moment that for technical reasons, it is more convenient to introduce the square root of $\varepsilon$ rather than $\varepsilon$ itself). It is also convenient to make the following change of variables:

$$
\tilde{V}:=\sqrt{\varepsilon} V ; \tilde{P}:=\varepsilon P .
$$

The cost function being nonnegative and positively homogeneous of degree 2 , the equivalent problem obtained after this change of variables can be written as

$\left(\tilde{\mathcal{P}}_{\varepsilon}\right) \quad \operatorname{Min}_{\tilde{V}, \tilde{P}} \frac{1}{2} \tilde{V}^{t} Z \tilde{V} ; Z \tilde{V}-\tilde{P} / \tilde{V}=0 ; \tilde{V}_{0}=V^{R} ; V^{b} \leq \tilde{V} \leq V^{\sharp} ; \varepsilon P^{b} \leq \tilde{P} \leq \varepsilon P^{\sharp}$.

We call $\left(\tilde{V}^{\varepsilon}, \tilde{P}^{\varepsilon}\right)$ a possible solution of $\left(\tilde{\mathcal{P}}_{\mathcal{\varepsilon}}\right)$. We observe that, except for the bound constraints on $\tilde{P}$ in which $\varepsilon$ appears, this new problem is identical to $(\tilde{\mathcal{P}})$. From a mathematical point of view, it is equivalent to make the voltage go to infinity, with a fixed range of delivered power, or to make the power go to 0 , with a fixed range of voltage. In other words, the high voltage approximation is nothing but a small power approximation.

By elementary case we mean the following situation ${ }^{2}: V^{b}=-\infty$ and $V^{\sharp}=+\infty$ over the network, i.e., there is no bound constraints on the voltage. This is a simple situation where various hypotheses can be easily checked.

The limit problem, obtained for $\varepsilon=0$, is

$$
\operatorname{Min}_{\tilde{V}, \tilde{P}} \frac{1}{2} \tilde{V}^{t} Z \tilde{V} ; Z \tilde{V}-\tilde{P} / \tilde{V}=0 ; \tilde{V}_{0}=V^{R} ; V^{b} \leq \tilde{V} \leq V^{\sharp} ; 0 P^{b} \leq \tilde{P} \leq 0 P^{\sharp},
$$

\footnotetext{
${ }^{2}$ Sometimes we write an equality between a vector and a scalar value: this means that each component of the vector is equal to the scalar value.
} 
where for writing the bound constraints on $\tilde{P}$, we use the convention

$$
0 \times(-\infty)=-\infty, 0 \times(+\infty)=+\infty
$$

and $0 P^{b}$ (resp. $\left.0 P^{\sharp}\right)$ is the $n$-dimensional vector with $k$-th component $0 \times P_{k}^{b}\left(\right.$ resp. $\left.0 \times P_{k}^{\sharp}\right)$. The physical motivation for designing high voltage networks is to reduce the currents, hence the power lost within the lines. In order to achieve this, the bound constraints on the voltage must not forbid values that are close to the reference value, so that we assume that $V^{b} \leq V^{R} \leq V^{\sharp}$. Also, if $k \in \mathcal{S}$ is such that $V_{k}^{b}=V^{R}=V_{k}^{\sharp}$, then we may identify node $k$ with node 0 . Consequently, we assume that

$$
V^{b} \leq V^{R} \leq V^{\sharp} \text { and } V^{b}<V^{\sharp},
$$

where the strict inequality between vectors means strict inequality between all components.

LEMMa 3.1. Assume that $(H 1)$ holds. Then the limit problem has a unique solution $\left(\tilde{V}^{0}, \tilde{P}^{0}\right)$ defined as follows:

$$
\tilde{V}^{0}=V^{R} \text { and } \tilde{P}^{0}=0 .
$$

Proof. We first check that $\left(\tilde{V}^{0}, \tilde{P}^{0}\right)$ defined as above is feasible for $\left(\tilde{\mathcal{P}}_{0}\right)$. As $\tilde{V}^{0}$ is constant over the network, we have $Z \tilde{V}^{0}=0$. Because $\tilde{V}^{0}=V^{R}>0$, the term $\tilde{P}^{0} / \tilde{V}^{0}$ is well defined and has value 0 . Therefore the power equation is satisfied. The bound constraints are also satisfied, thanks to $(H 1)$.

Now $\left(\tilde{V}^{0}, \tilde{P}^{0}\right)$ is associated with a null cost. The cost function being non negative, $\left(\tilde{V}^{0}, \tilde{P}^{0}\right)$ is solution of $\left(\tilde{\mathcal{P}}_{0}\right)$. Any other solution $(V, P)$ is also associated with a null cost, hence must satisfy $Z V=0$. It follows that $V=\tilde{V}^{0}$, and we deduce from the power equation that $P=\tilde{P}^{0}$ as well.

4. Mathematical tools. This self-contained section presents the mathematical tools that we need from the perturbation theory for nonlinear programming. It is devoted to the presentation of two known results, and to the derivation of a third one. The first result gives the second-order expansion of the cost and the first-order expansion of the solution under weak hypotheses. The second one needs much stronger hypotheses, but gives the analytic expansion of the cost and solution. Combining these two results, we obtain the analytic expansion of the cost and solution under weaker hypotheses than for the second result.

We consider an abstract finite-dimensional nonlinear optimization problem

$$
\operatorname{Min}_{x \in \mathbb{R}^{n}} f(x, \varepsilon) ; g(x, \varepsilon) \prec 0
$$

where $f: \mathbb{R}^{n} \times \mathbb{R} \rightarrow \mathbb{R}$ and $g: \mathbb{R}^{n} \times \mathbb{R} \rightarrow \mathbb{R}^{p}$ are $C^{2}$ mappings, and where the symbol $\prec$ stands for a finite number of equalities and inequalities, namely for a given $q \in\{1, \ldots, p\}$ :

$$
z \prec 0 \text { iff } z_{i}=0, i=1, \ldots, q \text {, and } z_{j} \leq 0, j=q+1, \ldots, p \text {. }
$$


We consider $\left(P_{0}\right)$ as the unperturbed problem, and $\varepsilon \geq 0$ as the perturbation parameter. This perturbation parameter is assumed to be scalar, and our aim is to compute the expansion of the cost and solution of the problem.

We assume in this section that $\left(P_{0}\right)$ has a unique solution $x^{0}$, and that for $\varepsilon>0$ small enough, the set of solutions of $\left(P_{\varepsilon}\right)$ is non empty and uniformly bounded. This kind of condition can be checked by ad hoc conditions on specific examples.

We denote the set of active inequality constraints, the Lagrangian function associated with $\left(P_{\varepsilon}\right)$, and the set of Lagrange multipliers associated with $x^{0}$ as, respectively

$$
\begin{aligned}
I(x, \varepsilon) & :=\left\{j=q+1, \cdots, p ; g_{j}(x, \varepsilon)=0\right\}, \\
L(x, \lambda, \varepsilon) & :=f(x, \varepsilon)+\lambda^{t} g(x, \varepsilon), \\
\Lambda & :=\left\{\lambda \in \mathbb{R}^{p} ; L_{x}^{\prime}\left(x^{0}, \lambda, 0\right)=0 ; \lambda_{j} \geq 0, \lambda_{j} g_{j}\left(x^{0}, 0\right)=0, j>q\right\} .
\end{aligned}
$$

An important condition of qualification, due to Mangasarian and Fromovitz [12], is the following:

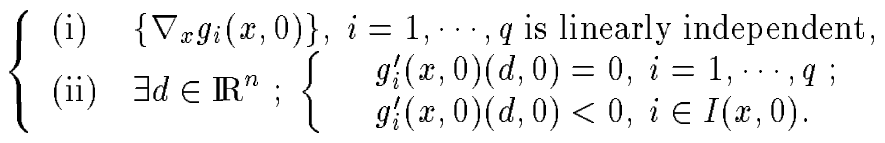

This hypothesis is satisfied iff the set of Lagrange multiplier is non empty and bounded [9] and is also equivalent to a certain stability property of the feasible set [13]. It seems therefore natural to assume it in order to conduct a perturbation analysis. However, in the specific application we have in mind, $(M F)$ is not satisfied. Consequently we will rely on the directional constraint qualification due to [11]

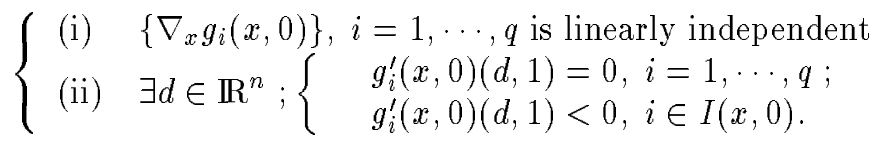

It is easily checked that $(M F) \Rightarrow(D Q)$. The next step consists in linearizing the data w.r.t. $(x, \varepsilon)$ at $\left(x^{0}, 0\right)$. Denoting by $(d, 1)$ the direction, we get the linearized problem

$$
\begin{cases}\operatorname{Min}_{x \in \mathbb{R}^{n}} f^{\prime}\left(x^{0}, 0\right)(d, 1) ; & g_{i}^{\prime}(x, 0)(d, 1)=0, i=1, \cdots, q ; \\ & g_{i}^{\prime}(x, 0)(d, 1) \leq 0, i \in I(x) .\end{cases}
$$

A nice interpretation of condition $(D Q)$ is that it is equivalent to the condition of Mangasarian and Fromovitz for the linearized problem. It follows that the dual of $(\mathcal{L})$, whose expression is

$$
\operatorname{Max} L_{\varepsilon}^{\prime}\left(x^{0}, \lambda, 0\right) ; \lambda \in \Lambda
$$

has, if $\Lambda$ is non empty, a nonempty and bounded set of solution $S(\mathcal{D})$. 
We need some second-order analysis. The critical cone is defined as

$$
\begin{aligned}
C(x):=\left\{d \in \mathbb{R}^{n} ; f_{x}^{\prime}(x, 0) d \leq 0 ; \quad\right. & g_{i}^{\prime}(x, 0)(d, 0)=0, i=1, \cdots, q \\
& \left.g_{i}^{\prime}(x, 0)(d, 0) \leq 0, i \in I(x, 0)\right\} .
\end{aligned}
$$

The directional second-order condition, due to [15], is as follows:

$$
\sup _{\lambda \in S(\mathcal{D})} L_{x^{2}}^{\prime \prime}\left(x^{0}, \lambda, 0\right) d d>0, \forall d \in C\left(x^{0}\right) \backslash\{0\}
$$

Note that, the supremum over an empty set being $-\infty$, the strong second-order condition implies that the set of multipliers in nonempty (except in the special case $C\left(x^{0}\right)=\{0\}$, but it is also true in this case that the set of multipliers in nonempty).

We say that a map $\mathbb{R}_{+} \rightarrow \mathbb{R}^{n}, \varepsilon \rightarrow x^{\varepsilon}$, is a path if $x^{\varepsilon} \rightarrow x^{0}$ when $\varepsilon \downarrow 0$. A path of $\left(\varepsilon^{2}\right)$-solutions is a path $x^{\varepsilon}$ such that, for $\varepsilon>0$ small enough, $x^{\varepsilon}$ is feasible for $\left(P_{\varepsilon}\right)$ and $f\left(x^{\varepsilon}, \varepsilon\right) \leq v\left(P_{\varepsilon}\right)+o\left(\varepsilon^{2}\right)$. We say that $d \in \mathbb{R}^{n}$ is a first-order term associated with a path $x^{\varepsilon}$ if $d$ is a limit-point of $\left(x^{\varepsilon}-x^{0}\right) / \varepsilon$.

We define the following subproblem

$$
\operatorname{Min}_{d \in S(\mathcal{L})} \max _{\lambda \in S(\mathcal{D})} L^{\prime \prime}\left(x^{0}, \lambda, 0\right)(d, 1)(d, 1) .
$$

As $S(\mathcal{L})$ is a polyhedron, if $S(\mathcal{D})$ is a singleton, then $(Q)$ is a quadratic optimization problem (a problem with quadratic cost and linear constraints).

The result below is due to [2] and [6], and has its root in [15].

TheOREM 4.1. Assume that the directional constraint qualification hypothesis is satisfied, as well as the directional second-order condition. Then

(i) (Stability) any path $x^{\varepsilon}$ of $o\left(\varepsilon^{2}\right)$ solutions satisfies $x(\varepsilon)=x^{0}+O(\varepsilon)$.

(ii) (Expansion of solutions) The union of all first-order terms associated with $o\left(\varepsilon^{2}\right)$ solutions is equal to $S(Q)$. In particular, if $S(Q)=\{\bar{d}\}$, then a path of exact solution $x^{\varepsilon}$ satisfies $x^{\varepsilon}=x^{0}+\varepsilon \bar{d}+o(\varepsilon)$.

We now quote a second abstract result, based on stronger hypotheses. The condition of linear independence (of gradients of active constraints at $x^{0}$ ) is

$$
\left\{\nabla_{x} g_{i}\left(x^{0}, 0\right) ; i \in\{1, \cdots, q\} \cup I\left(x^{0}, 0\right)\right\} \text { is linearly independent } .
$$

This condition implies that with $x^{0}$ is associated a unique multiplier $\lambda^{0}$. We defined the enlarged critical cone as

$$
C^{\sharp}\left(x^{0}, \lambda^{0}\right):=\left\{d \in \mathbb{R}^{n} ; g_{i}^{\prime}\left(x^{0}, 0\right)(d, 0)=0, i \in\{1, \cdots, q\} \cup\left\{j \in I\left(x^{0}, 0\right) ; \lambda_{j}>0\right\}\right\} .
$$

It is easily checked that $C\left(x^{0}\right) \subset C^{\sharp}\left(x^{0}\right)$. Both sets coincide if $\left(x^{0}, \lambda^{0}\right)$ is a strictly complementary pair, in the sense that $\lambda_{j}>0$ whenever $g_{j}\left(x^{0}, 0\right)=0$, for all $j \in I\left(x^{0}, 0\right)$. 
The strong second-order condition, due to [14] assumes that with $x^{0}$ is associated a unique multiplier $\lambda^{0}$ such that

$$
L_{x^{2}}^{\prime \prime}\left(x^{0}, \lambda^{0}, 0\right) d d>0, \forall d \in C^{\sharp}\left(x^{0}, \lambda^{0}\right) \backslash\{0\} .
$$

The statement below is due to [7].

THEOREM 4.2. Assume that $\left(x^{0}, \lambda^{0}\right)$ satisfy the condition of linear independence as well as the strong second-order condition. Then in a certain neighborhood of $x^{0}$, for $\varepsilon>0$ small enough, $\left(P_{\varepsilon}\right)$ has a unique solution $x^{\varepsilon}$ and the mapping $\varepsilon \rightarrow\left(x^{\varepsilon}, \lambda^{\varepsilon}, v\left(P_{\varepsilon}\right)\right)$ is (real) analytical. The coefficients of the expansion of $x^{\varepsilon}$ and $\lambda^{\varepsilon}$ can be computed by expanding the optimality system as in [7]. The first order expansion is $x^{\varepsilon}=x^{0}+\varepsilon \bar{d}+O\left(\varepsilon^{2}\right)$, where $\bar{d}$ is the unique solution of $S(Q)$.

We now combine the above two results in order to deduce a third one. We say that the directional linear independence qualification condition holds if $(\mathcal{L})$ is feasible and satisfies the hypothesis of linear independence at each solution of $(Q)$. We say that the strong directional second-order condition holds if $(\mathcal{D})$ has a unique solution $\lambda^{0}$, such that $(6)$ holds.

THEOREM 4.3. Assume that $x^{0}$ is a local solution of $\left(P_{0}\right)$, satisfying

(i) the directional linear independence qualification condition,

(i) the strong directional second-order condition. Then the conclusion of Theorem 4.2 holds.

Proof. By (i), the linearized problem is qualified, i.e. $(D Q)$ holds. Condition (ii) implies the directional second-order condition (which therefore involves no other multiplier than $\lambda^{0}$ ). We may apply Theorem 4.1. By (ii), problem $(Q)$ has a unique solution $\bar{d}$. Let us denote by $I^{*}$ the set of active inequality constraints for $(\mathcal{L})$, associated with $\bar{d}$. Let $x^{\varepsilon}>0$ be a path of solutions. The inequality constraints in $I \backslash I^{*}$ are not active for $\varepsilon>0$ small enough. Therefore, $x^{\varepsilon}$ is a local solution of

$$
\operatorname{Min}_{x \in \mathbb{R}^{n}} f(x, \varepsilon) ; g_{i}(x, \varepsilon)=0, i=1, \cdots, q ; g_{i}(x, \varepsilon) \leq 0, i \in I^{*} .
$$

Now $x^{0}$ is a local solution of $\left(\hat{P}_{0}\right)$ associated with a unique multiplier $\lambda^{0}$ (as can be checked with the second-order sufficient conditions) and we may apply Theorem 4.2 to problem $\left(\hat{P}_{0}\right)$ in order to get the conclusion. $\mathrm{Q}$

5. Study of the limit problem. Let us now come back to the optimal DC power flow problem. We observe that the limit problem is in general not qualified, in the sense that $(M F)$ does not hold. Indeed, if there exists $k \in \mathcal{S}$ such that both $P_{k}^{b}$ and $P_{k}^{\sharp}$ have finite but different values, then we get the constraint $0 \leq \tilde{P}_{k} \leq 0$, and these two inequalities cannot be strictly satisfied.

We need the following notation for the active constraints: $\bar{V}^{b}, \bar{V}^{\sharp}$ are $n$-dimensional vectors defined by

$$
\bar{V}_{k}^{b}=V_{k}^{b} \text { if } V_{k}^{b}=V^{R},-\infty \text { if not } ; \bar{V}_{k}^{\sharp}=V_{k}^{\sharp} \text { if } V_{k}^{\sharp}=V^{R},+\infty \text { if not, } \forall k \in \mathcal{S} \text {. }
$$


Linearizing the data of $\left(\tilde{\mathcal{P}}_{\varepsilon}\right)$ with respect to $(\tilde{V}, \tilde{P}, \varepsilon)$ at $\left(\tilde{V}^{0}, \tilde{P}^{0}, 0\right)$ we obtain the linearized problem

$$
\begin{cases}\operatorname{Min}_{d V, d P}\left(\tilde{V}^{0}\right)^{t} Z d V ; \quad & Z^{R} d V=d P / \tilde{V}^{0} ; d V_{0}=0 ; \\ & \bar{V}^{b} \leq V^{R}+d V \leq \bar{V}^{\sharp} ; \quad P^{b} \leq d P \leq P^{\sharp} .\end{cases}
$$

As $\left(\tilde{V}^{0}\right)^{t} Z d V=\left(Z \tilde{V}^{0}\right)^{t} d V=0$, we get the equality $S(\mathcal{L})=F(\mathcal{L})$. In the Lemma below, we use hypothesis $(H 1)$ defined just before Lemma 3.1. Denote by $\mathcal{S} \overline{\bar{P}}$ the set of nodes over which the lower and upper bound on $\tilde{P}$ are equal. In order to apply the abstrac result, we view the bound constraints over $\tilde{P}$ as equalities over $\mathcal{S}_{\bar{P}}^{\bar{y}}$.

Lemma 5.1. Assume that ( $H 1)$ holds. Then:

(i) The gradient of equality constraints are linearly independent.

(ii) The limit problem is directionally qualified iff there exists $(d V, d P) \in F(\mathcal{L})$, with each component strictly between the bounds whenever they are not equal.

(iii) If the limit problem is directionally qualified, then $(\mathcal{D})$ has a unique solution, namely the null multiplier.

Proof. (i) Checking (i) amounts to check that the linearized equality constraints are onto, i.e., the system

$$
d V_{0}=W^{0} ; Z d V-\frac{d P}{V}=W^{1} \text { over } \mathcal{S} ; d P=W^{2} \text { over } \mathcal{S}_{\bar{P}}^{=},
$$

has (at least one) solution for any $\left(W^{0}, W^{1}, W^{2}\right)$. Set $d P$ to 0 over $\mathcal{S} \backslash \mathcal{S} \overline{\bar{P}}$. It remains to solve

$$
d V_{0}=W^{0} ; Z d V=\frac{d P}{V}+W^{1}
$$

which is the equation of a linear DC network with given voltage at node 0 and given inputs of current at other nodes: this problem has a unique solution.

(ii) This is an immediate consequence of point (i).

(iii) As the gradient of the cost of problem $\tilde{\mathcal{P}}_{0}$ is null, the set of Lagrange multipliers is a cone. Problem $(\mathcal{D})$ consists in maximizing a linear cost over this cone. It follows that $S(\mathcal{D})$ is itself a cone. We know that if directional qualification holds, then the set of solutions is nonempty and bounded. Being a cone, this set can be nothing else than $\{0\}$. $D$

Remark In the elementary case (unconstrained voltage) point (i) of the above Lemma is satisfied. Therefore, the limit problem is directionally qualified. Another situation where directional qualification can be checked is when the upper bound on the voltage are strictly greater than $V^{R}$, and there is no upper bound on the power. It follows that problem $(\mathcal{L})$ has no upper bound. Now we can take $d P=\alpha(1, \cdots, 1)$, with $\alpha>0$ large enough to obtain $d P>P^{b}$. Then $d V$, solution of the linearized power equation is positive, hence $(d V, d P)$ satisfies the condition for directional qualification.

We may have a better insight on these qualification conditions by making an analogy with the optimal control theory. See $d P$ as the control and $d V$ as the state. What we called 
the elementary case is when there is no state constraint. In the case of one-sided constraints for the control and state, where we have taken advantage of the positivity of the mapping control $\rightarrow$ state, the discussion parallels the one for the control of nonlinear elliptic equations $[5]$.

We assume in the sequel that the directional qualification hypothesis holds. As $S(\mathcal{D})=$ $\{0\}$, the cost of the quadratic subproblem reduces in our application to the Hessian of the cost. Using $S(\mathcal{L})=F(\mathcal{L})$, we can reformulate this problem as

$$
\operatorname{Min}_{d V, d P} \frac{1}{2}(d V)^{t} Z d V ;(d V, d P) \in F(\mathcal{L})
$$

Because $d V_{0}$ is set to 0 , the system $Z d V=d P / \tilde{V}^{0}$ has a unique solution $d V=$ $Z^{-1}\left(d P / \tilde{V}^{0}\right)$. Introducing the auxiliary variable $h:=d P / \tilde{V}^{0}$, and substituting in $(S P)$, we obtain the equivalent problem

$$
\operatorname{Min}_{h} \frac{1}{2} h^{t} Z^{-1} h ; d V_{0}=0 ; \bar{V}^{b} \leq Z^{-1} h \leq \bar{V}^{\sharp} ; \frac{P^{b}}{V^{0}} \leq h \leq \frac{P^{\sharp}}{V^{0}} .
$$

This problem has a unique solution, namely the projection, along the norm associated with $Z^{-1}$, of the origin over the feasible set. We call $(\overline{d V}, \overline{d P})$ the solution of $(S P)$.

The following Lemma allows to check the second-order conditions.

Lemma 5.2. Consider the limit problem $\left(\tilde{\mathcal{P}}_{0}\right)$. Then the Hessian of the cost is positive definite over the space tangent to the equality constraints.

Proof. The quadratic form $(d V)^{t} Z d V$ is positive semi-definite, and its null space is the kernel of $Z$, which is known to be space of vectors with all equal components. As $d V_{0}=0$, it follows that $(d V)^{t} Z d V>$ unless $d V=0$. In the latter case, $d P=0$ whenever the linearized power equation is satisfied. As a consequence, if $(d V, d P)$ is tangent to the linearized equality constraints, the quadratic form associated with the Hessian of the cost is positive unless $(d V, d P)=0$, as was to be proved. $\mathrm{Q}$

6. Expansion of the solution and multipliers. We start with a technical Lemma, allowing to check some of the hypotheses of the results of section 4 .

LEMMa 6.1. The set of solutions of $\left(\tilde{\mathcal{P}}_{\varepsilon}\right)$ is, for $\varepsilon$ small enough, non empty and uniformly bounded.

Proof. By directional qualification, we know that

$$
v\left(\tilde{\mathcal{P}}_{\varepsilon}\right) \leq v\left(\tilde{\mathcal{P}}_{0}\right)+\varepsilon v(\mathcal{L})+o(\varepsilon) \leq O(\varepsilon) .
$$

Due to the condition $\tilde{V}_{0}=V^{R}$, the cost function of $v\left(\tilde{\mathcal{P}}_{\varepsilon}\right)$ satisfies for some $\alpha>0$ the relation $\frac{1}{2} V^{t} Z V \geq \alpha\left\|V-V^{R}\right\|^{2}$. Denoting by . the componentwise multiplication of vectors,we have $\|P\|=\|V \cdot Z V\|=O\left(\left\|V-V^{R}\right\|^{2}\right)$. From these relations, we deduce for $\varepsilon$ small enough the uniform boudedness of minimizing sequences. In addition, if $\left(V^{k}, P^{k}\right)$ is a minimizing 
sequence, from $\left\|V^{k}-V^{R}\right\|^{2} \leq O(\varepsilon)$, we deduce (for $k$ large enough and $\varepsilon$ small enough) that the limit points of $V^{k}$ have positive values. Consequently, these limit points are solution of $\left(\tilde{\mathcal{P}}_{\varepsilon}\right)$. As the same estimates hold for the limit points, the conclusion follows.

Theorem 6.2. Assume $(H 1)$ and $\left(\tilde{\mathcal{P}}_{0}\right)$ to be directionally qualified. Then, for $\varepsilon$ small enough, $\left(\tilde{\mathcal{P}}_{\varepsilon}\right)$ has a unique solution $\left(\tilde{V}^{\varepsilon}, \tilde{P}^{\varepsilon}\right)$, and the following expansions hold:

$$
\begin{aligned}
v\left(\tilde{\mathcal{P}}_{\varepsilon}\right) & =v\left(\tilde{\mathcal{P}}_{0}\right)+\varepsilon^{2} v(S P)+o\left(\varepsilon^{2}\right), \\
\left(\tilde{V}^{\varepsilon}, \tilde{P}^{\varepsilon}\right) & =\left(\tilde{V}^{0}, \tilde{P}^{0}\right)+\varepsilon(\overline{d V}, \overline{d P})+o(\varepsilon) .
\end{aligned}
$$

With $\left(\tilde{V}^{\varepsilon}, \tilde{P}^{\varepsilon}\right)$ is associated a non-empty and uniformly bounded set of multipliers $\Lambda_{\varepsilon}$. This set of multipliers converges to 0 , in the sense that if $\lambda^{\varepsilon} \in \Lambda_{\varepsilon}$, and $\varepsilon \downarrow 0$, then $\lambda^{\varepsilon} \rightarrow 0$.

Proof. Apply Theorem 4.1, using Lemmas 5.1, 5.2 and 6.1.

We now study the higher-order expansion of the solution of $\left(\tilde{\mathcal{P}}_{\varepsilon}\right)$ and associated multiplier. We observe that the result of [7] cannot be used directly, because problem $\left(\tilde{\mathcal{P}}_{0}\right)$ is not in general qualified, as already observed. However, we may apply Theorem 4.3, if the hypothesis below holds.

(H2) Linear independence of gradients of active constraint for $(\mathcal{L})$ at $(\overline{d V}, \overline{d P})$.

Let us state first a natural sufficient condition for $(H 2)$.

Lemma 6.3. Assume that at no node of the network, the bound constraints on $\overline{d V}$ and $\overline{d P}$ are both active. Then $(H 2)$ holds.

Proof. Let the bound constraints on the voltage (resp. power) be active on $\mathcal{S}_{V}^{a}$ (resp. $\left.\mathcal{S}_{P}^{a}\right)$. By hypothesis, $\mathcal{S}_{V}^{a} \cap \mathcal{S}_{P}^{a}=\phi$. Set $d P$ to 0 over $\mathcal{S} \backslash\left(\mathcal{S}_{V}^{a} \cup \mathcal{S}_{P}^{a}\right)$. We must check that it is possible to solve a linear DC problem with voltage fixed over $\mathcal{S}_{V}^{a}$ as well as the reference node, and the injection of current fixed over the other nodes. This problem is known to have a unique solution. $\mathrm{Q}$

THEOREM 6.4. Assume that (H1) and (H2) hold. Then the mapping $\varepsilon \rightarrow\left(\tilde{V}^{\varepsilon}, \tilde{P}^{\varepsilon}, \lambda^{\varepsilon}\right)$, where $\left(\tilde{V}^{\varepsilon}, \tilde{P}^{\varepsilon}\right) \in S\left(\tilde{\mathcal{P}_{\varepsilon}}\right)$ and $\lambda^{\varepsilon}$ is the multiplier associated with the power equation is, for $\varepsilon$ small enough, analytical.

Proof. By (H2), Lemmas 5.1, 5.2 and 6.1, the hypotheses of Theorem 4.3 are satisfied. The conclusion follows.

7. Back to the physical problem. In this section we come back to the physical variables $\left(V^{\varepsilon}, P^{\varepsilon}\right)$ and give simple interpretations of the above results. Restating Theorem 6.2 , we obtain

Theorem 7.1. Assume $\left(\tilde{\mathcal{P}}_{0}\right)$ to be directionally qualified. Then, for $\varepsilon$ small enough, $\left(\mathcal{P}_{\varepsilon}\right)$ has a unique solution $\left(V^{\varepsilon}, P^{\varepsilon}\right)$, and the following expansions hold:

$$
v\left(\mathcal{P}_{\varepsilon}\right)=\varepsilon v(S P)+o(\varepsilon),
$$

$\mathrm{RR} \mathrm{n}^{\circ} 2408$ 


$$
\begin{aligned}
V^{\varepsilon} & =\frac{1}{\sqrt{\varepsilon}} V^{R}+\sqrt{\varepsilon} \overline{d V}+o(\sqrt{\varepsilon}), \\
P^{\varepsilon} & =\overline{d P}+o(\varepsilon) .
\end{aligned}
$$

In particular, we deduce that when the nominal value is very high, and if the bound constraints are compatible, in the sense that $(H 1)$ holds, then

(a) the loss of power is of the order of the square of the inverse of the average value of the network,

(b) the difference between the average value and the actual value of the voltage is of the order of the inverse of the average value,

(c) the distribution of power over the network has a limit.

Concluding Remark There are two possible uses of our technical results. The first one is the above set of qualitative remarks (a) to (c), which are of interest by themself. The second possibility is to use the first-order expansion as the starting point of a numerical algorithm, dedicated to a quantitative resolution of the optimal power flow problem. Still, the most important aspect of the result is that it suggests a possible extension to the $\mathrm{AC}$ power flow problem, whose importance was stressed in the introduction.

\section{REFERENCES}

[1] J.P. Aubin, P.A. Raviart, On the resolution of equations arising in load flow problems, Proc. IEEE Power Industry Computer Applications Conf., 1965, pp. 119-132.

[2] A. Auslender and R. Cominetti, First and second order sensitivity analysis of nonlinear programs under directional constraint qualification conditions, Optimization 21(1990), pp. 351-363.

[3] G. Blanchon, J.F. Bonnans, J.C. Dodu, Optimisation de réseaux électriques de grande taille. Lect. Notes Inf. Cont. Sci. 144, Springer Verlag, Berlin, 1990, pp. 423-431.

[4] J.F. Bonnans: Rapport de Contrat EDF-INRIA, 1994.

[5] J.F. Bonnans, E. Casas: Contrôle de systèmes elliptiques semilinéaires comportant des contraintes sur l'état, in "Nonlinear partial differential equations and their applications, Collège de France seminar vol. VIII", H. Brézis \& J.L. Lions eds., Pitman Research Notes in Mathematics Series 166, Longman Scientific \& Technical, New York, 1988, pp. 69-86.

[6] J.F. Bonnans, A.D. Ioffe and A. Shapiro, Développement de solutions exactes et approchées en programmation non linéaire. Comptes Rendus Acad. Sci. Paris, t. 315, Série I, pp. 119-123.

[7] J.F. Bonnans, A. Sulem (1993): Pseudopower expansion of solutions of generalized equations and constrained optimization problems. Rapport de Recherche INRIA 1956. Math. Programming, to appear.

[8] C. Carpentier, CRIC: a new active-reactive decoupling process in load flows, optimal power flows and system control, in Proc. IFAC Conf. Power Systems and Power Plant Control, Beijing, 1986, pp. 65-70.

[9] J. Gauvin, A necessary and sufficient regularity condition to have bounded multipliers in nonconvex programming, Math. Programming 12(1977), pp. 136-138.

[10] J. Gauvin and R. Janin, Directional behavior of optimal solutions in nonlinear mathematical programming, Math. Operations Research 13(1988), pp. 629-649.

[11] B. Gollan, On the marginal function in nonlinear programming, Math. Oper. Res. 9(1984), pp. 208-221. 
[12] O.L. Mangasarian and S. Fromovitz, The Fritz John necessary optimality conditions in the presence of equality and inequality constraints, J. Math. Analysis Appl. 17(1967), pp. 37-47.

[13] S.M. Robinson, Stability theory for systems of inequalities, part II: differentiable nonlinear systems, SIAM J. Numer. Analysis 13(1976), pp. 497-513.

[14] S.M. Robinson, Strongly regular generalized equations, Math. Operations Research 5(1980), pp. 43-62.

[15] A. Shapiro, Sensitivity analysis of nonlinear programs and differentiability properties of metric projections, SIAM J. Control \& Opt. 26(1988), pp. 628-645. 
Unité de recherche INRIA Lorraine, Technopôle de Nancy-Brabois, Campus scientifique, 615 rue du Jardin Botanique, BP 101, 54600 VILLERS LES NANCY

Unité de recherche INRIA Rennes, Irisa, Campus universitaire de Beaulieu, 35042 RENNES Cedex Unité de recherche INRIA Rhône-Alpes, 46 avenue Félix Viallet, 38031 GRENOBLE Cedex 1

Unité de recherche INRIA Rocquencourt, Domaine de Voluceau, Rocquencourt, BP 105, 78153 LE CHESNAY Cedex

Unité de recherche INRIA Sophia-Antipolis, 2004 route des Lucioles, BP 93, 06902 SOPHIA-ANTIPOLIS Cedex

\section{Éditeur}

INRIA, Domaine de Voluceau, Rocquencourt, BP 105, 78153 LE CHESNAY Cedex (France)

ISSN 0249-6399 